\title{
Importance of Microwave Heating in Organic Synthesis
}

\author{
Sumitra Nain a, Ruchi Singh a, Subramanian Ravichandran (iD b,*
}

\begin{tabular}{l} 
A R T I C L E I N F O \\
\hline Received: 13 December 2018 \\
Revised: 2 January 2019 \\
Accepted: 16 January 2019 \\
Available online: 27 January 2019 \\
KE Y W O R D S
\end{tabular}

Microwave heating

Organic synthesis

Chemical reactions

\section{A B S T R A C T}

Microwave heating is widely used as a convenient source of heating in organic synthesis. The heating is instantaneous and very specific. Nowadays, Microwave-assisted organic synthesis could be considered in which all of the previously heated reactions could be performed using this technique. The benefits of microwave-assisted organic synthesis are increasingly making the technique more widely established. The technique offers simple, clean, fast, efficient, and economic features for the synthesis of a large number of organic molecules. In the present article, an attempt was made to focus on the importance of the microwave heating.

\section{GRA PH ICALABSTRACT}

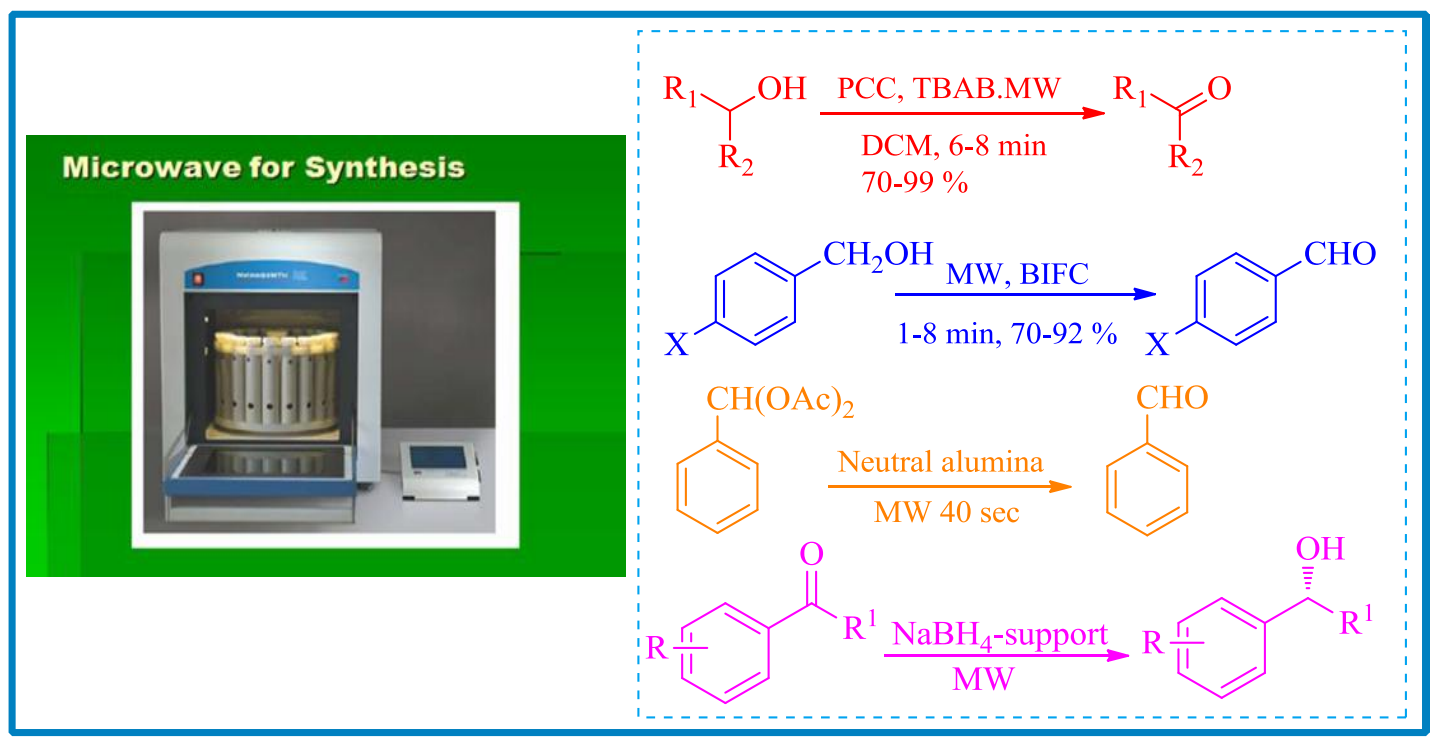

\footnotetext{
* Corresponding author's E-mail address: ravichanduru@yahoo.com, Tel.: 00919840735064

a Department of Pharmacy, Banasthali University, Banasthali (Rajasthan), India -304 022.

${ }^{b}$ Associate Professor in Chemistry, Lovely Professional University, Jalandhar (Punjab), India-144 411.
} 


\section{Introduction}

Microwave chemistry is the science of applying microwave radiation to chemical reactions [1-10]. Microwave synthesis represents a major breakthrough in the synthetic chemistry methodology; a dramatic change in the way chemical synthesis is performed. Microwave-assisted organic synthesis has revolutionized organic synthesis. Microwave heating has thus been found to be a very convenient thermal source not only in the kitchen but also in a chemical laboratory.

Chemists have explored the possibility of the application of a conventional microwave oven to carry out chemical reactions. Since the first published reports on the use of microwave irradiation to carry out organic chemical transformations, more than 6000 articles have been published in this fast moving and exciting field [11-18]. The application of microwave irradiation to activate and accelerate organic reactions has taken a new dimension and has experienced exponential growth in the last twenty years. Microwave chemistry is becoming increasingly popular both in industry and in academia. Microwaves can accelerate the rate of reaction, provide better yields and higher purity, uniform and selective heating with lower energy usage, achieve greater reproducibility of reactions and help in developing convenient and cleaner synthetic routes. The main advantages [1930] of microwave assisted organic synthesis are:

Faster reaction: Based on experimental data it has been found that microwaveenhanced chemical reaction rates can be faster than those of conventional heating methods. The microwave can use higher temperatures than conventional heating system, and consequently the reactions are completed in few minutes instead of hours, for instance, synthesis of fluorescein, which usually takes about 10 hours by conventional heating methods, can be conducted in only 35 minutes by means of microwave heating.

Better yield and higher purity: Less formation of side products are observed using microwave irradiation, and the product is recovered in higher yield. Consequently, the purification step is faster and easier. For example, microwave synthesis of aspirin results in an increase in the yield of the reaction, from $85 \%$ to $97 \%$.

Energy saving: Heating by means of microwave radiation is a highly efficient process and results in significant energy saving. This is primarily because microwaves heat up just the sample and not the apparatus, and therefore energy consumption is less. 
Uniform and selective heating: In conventional heating, the walls of the oil bath get heated first, and then the solvent. As a result of this distributed heating in an oil bath, there is always a temperature difference between the walls and the solvent. In the case of microwave heating, only the solvent and the solute particles are excited, which results in uniform heating of the solvent. Selective heating is based on the principle that different materials respond differently to microwaves. Some materials are transparent whereas others absorb microwaves.

Green synthesis: Reactions conducted using microwaves are cleaner and more ecofriendly than conventional heating methods. Microwaves heat the compounds directly; therefore, usage of solvents in the chemical reaction can be reduced or eliminated. Synthesis without solvent, in which reagents are absorbed on mineral support, has a great potential as it offers an eco-friendly green protocol in synthesis. The use of microwaves has also reduced the amount of purification required for the end products of chemical reactions involving toxic-reagents.

Reproducibility: Reactions with microwave heating are more reproducible compared to the conventional heating because of uniform heating and better control of process parameters. The temperature of chemical reactions can also be easily monitored.

The routine synthetic transformations are now being carried out by microwave heating. Microwave-assisted synthesis provides clean synthesis with the advantage of enhanced reaction rates, higher yields, greater selectivity, and economic for the synthesis of a large number of organic molecules, have provided the momentum for many chemists to switch from conventional heating method to microwave assisted chemistry. Microwave-assisted synthesis is rapidly becoming the method of choice in modern chemical synthesis and drug discovery. Microwave assisted synthesis has revolutionized chemical synthesis. Small molecules can be built in a fraction of the time required by conventional methods. In conventional heating methods oil bath or hot plate are used as a source of heat to a chemical reaction. Microwave irradiation is widely used as a source of heating in chemical synthesis. The basic mechanisms observed in microwave-assisted synthesis are dipolar polarization and conduction. Conventional methods of organic synthesis usually need longer heating time, tedious apparatus setup, which result in higher cost of process and the excessive use of solvents/reagents. During these processes there are many problems of health and safety for workers in addition to the environmental problems 
caused by their use and disposition as waste. Microwave synthesis is considered as an important approach toward green chemistry, because this technique is more eco-friendly. Due to its ability to couple directly with the reaction molecule and by passing thermal conductivity leading to a rapid rise in the temperature, microwave irradiation has been used to improve many organic syntheses.

\section{Organic synthesis assisted by Microwaves}

Microwave heating for carrying out reactions on solids has also attracted considerable attention in recent years. For such 'dry media' reactions, solid supports such as alumina, silica, montmorillonite clay and zeolites have been investigated. Application of microwave irradiation in chemical synthesis involves its use in the acceleration of chemical synthesis. Microwave-enhanced synthesis results in faster reactions, higher yields, and increased product purity. In addition, due to the availability of high-capacity microwave apparatus, the yields of the experiments have now easily scaled up from milligrams to kilograms, without the need to alter reaction parameters. Microwave-assisted synthesis can be suitably applied to the drug discovery process. Microwave-assisted organic synthesis has been the foremost and one of the most researched applications of microwaves in chemical reactions. Literature survey reveals that scientists have successfully conducted a large range of organic reactions. The following examples are impressive and provide a good insight into the field of microwave assisted organic synthesis [31-40].

\section{$N$-Alkylation}

$N$-Alkylation under microwave irradiation using phase transfer catalysts occupy a unique place in organic chemistry. Bogdal and co-workers reported the synthesis of $N$ alkyl phthalimides using phthalimide, alkyl halides, potassium carbonate and TBAB; giving products in 45-98\% yields (Scheme 1) [41].

\section{R-Alkylation}

$R$-Alkylation under microwave irradiation using potassium carbonate and TBACl giving products in 65-88\% yields (Scheme 2) [42].

\section{Oxidation}

Chakraborty reported the oxidation of secondary alcohol and benzyl alcohols using phase transfer catalysts [43]. Oxidation of secondary alcohols to acetone derivatives was carried out using PCC, tetrabutylammonium bromide and dichloromethane under microwave irradiation (6 $\mathrm{min}$ ), products were isolated in $70-99 \%$ yields. Oxidation of benzyl 
Scheme 1. synthesis of $N$-alkyl phthalimides under microwave irradiation

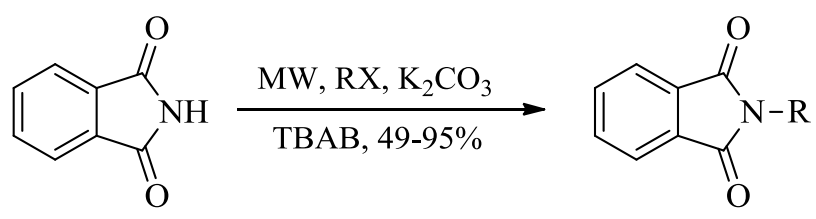

Scheme 2. $R$-Alkylation under microwave irradiation

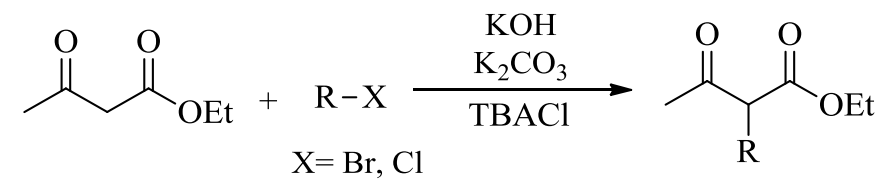

Scheme 3. Oxidation of secondary alcohols and benzyl alcohols using phase transfer catalysts under microwave irradiation
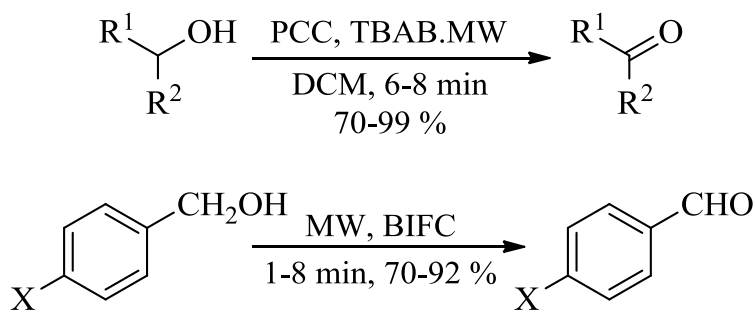

alcohols was conducted under microwave irradiation giving benzaldehyde derivatives in $70-92 \%$ yields (Scheme 3).

Oxidation of toluene with $\mathrm{KMnO}_{4}$ under normal conditions of refluxing takes 10-12 $\mathrm{hr}$ compared to reaction in microwave conditions, which takes only $5 \mathrm{~min}$ and the yield is $40 \%$ (Scheme 4) [46].

A Number of primary alcohols can be oxidized to the corresponding carboxylic acid using sodium tungstate as catalyst in $30 \%$ aqueous hydrogen peroxide (Scheme $5)$.

\section{Reduction}

Reduction of acetophenone with $\mathrm{NaBH}_{4}$ in microwave oven gives $92 \%$ yield of benzyl alcohol in 2 min (Scheme 6) [47].

\section{Knoevenagel Condensation}

Knoevenagel condensation is a well-known organic reaction, other applied in the synthesis of unsaturated acids, which are used as precursors for perfumes, flavonoids and as building blocks of many heterocycles. Gupta studied [48] knoevenagel condensation between carbonyl compounds and active methylene compounds, such as malonic acid, using tetrabutylammonium bromide, potassium carbonate in water forming unsaturated acids in excellent yield and purity under microwave irradiation(Scheme 7). 
Scheme 4. Oxidation of toluene with $\mathrm{KMnO}_{4}$ under microwave irradiation

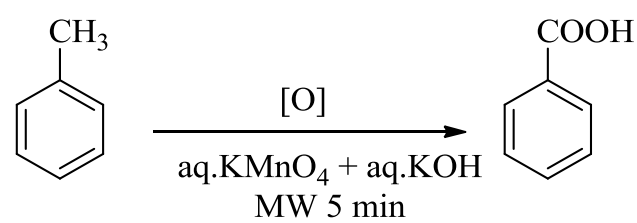

MW 5 min

Scheme 5. Synthesis of carboxylic acid using sodium tungstate

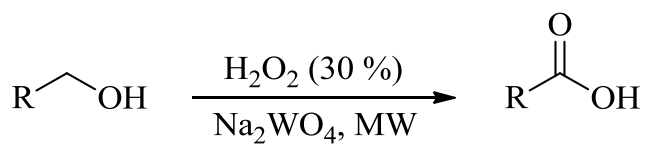

Scheme 6. Reduction of acetophenone with $\mathrm{NaBH}_{4}$ in microwave condition

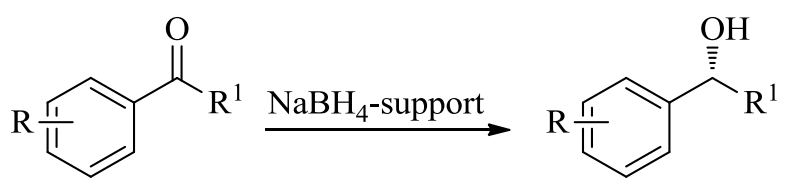

Scheme 7. Knoevenagel condensation under microwave irradiation

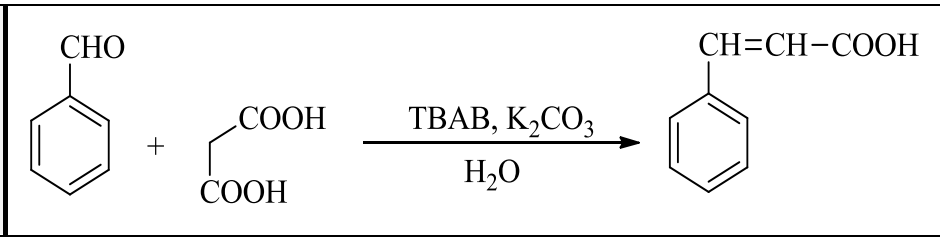

Scheme 8. Hydrolysis of benzyl chloride with water in microwave condition

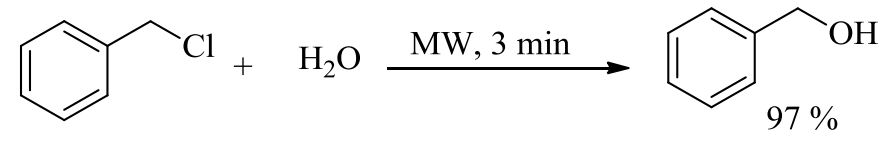

\section{Hydrolysis}

Hydrolysis of benzyl chloride with water in microwave oven gives $97 \%$ yield of benzyl alcohol in $3 \mathrm{~min}$ [49]. The usual hydrolysis in normal way takes about $35 \mathrm{~min}$ (Scheme 8). The usual hydrolysis of benzamide takes 1 hr. However, under microwave conditions, the hydrolysis is completed in 7 min giving $99 \%$ yield of benzoic acid (Scheme 9).

\section{Esterification}

A mixture of benzoic acid and n- propanol on heating in a microwave oven for $6 \mathrm{~min}$ in presence of catalytic amount of conc. Sulfuric acid gives propylbenzoate (Scheme 10) [50].

\section{Decarboxylation}

Conventional decarboxylation of carboxylic acids involve refluxing in quinoline in presence of copper chromate and the yields are low [51]. However, in the presence of 
microwaves decarboxylation takes place in much shorter time (Scheme 11).

\section{Deacetylation}

Aldehydes, phenol and alcohols are protected by acetylation. After the reaction, the deacetylation of the product is carried out usually under acidic or basic conditions the process takes long time and the yields are low. Use of microwave irradiation reduces the time of deacetylation and the yields are good (Scheme 12) [52].

\section{Mannich reaction}

A series of Mannich base derivatives were obtained expediently in good to excellent yields by microwave reaction (Scheme 13) [53]. In comparison with conventional results, results from microwave give better yields in shorter times.

Microwave-assisted organic synthesis is being widely applied in the pharmaceuticals industry, particularly for developing compounds in the lead optimization phase of drug discovery and development.
Scheme 9. hydrolysis of benzamide under microwave conditions

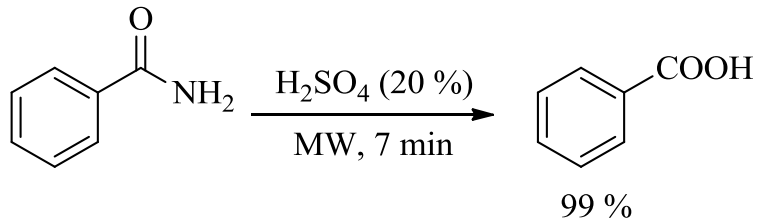

Scheme 10. Synthesis of propylbenzoate under microwave conditions

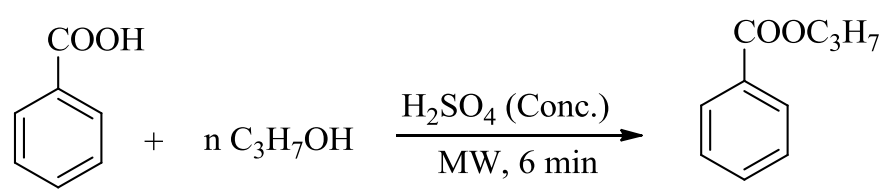

Scheme 11. Decarboxylation in the presence of microwaves

$$
\overbrace{\text { quinoline }}^{\longrightarrow}
$$

Scheme 12. Deacetylation under microwave irradiation

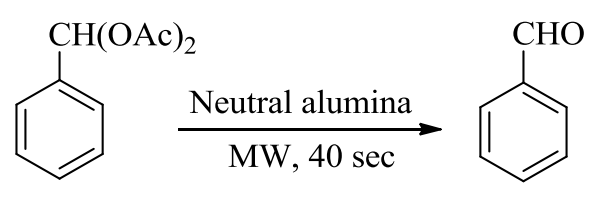


Scheme 13. Mannich reaction under
microwave irradiation

\section{Synthesis of Nanoparticles}

There are so many organic synthesis has been recently developed as these are solvent - free and assisted by microwave [41-60]. These synthesis are environmentally friendly, combines various reactions with solvent associated with selectivity and excluding organic solvents in waste free procedures. Microwave assisted synthesis is the promising technique in comparison to other methods existing for the synthesis of nanoparticles. Compared to conventional methods, microwave assisted synthesis was faster and provided particles with an average particle size of $12 \mathrm{~nm}$. Nanostructures with smaller sizes, narrower size distributions, and a higher degree of crystallization were obtained under microwave heating than those in conventional oil-bath heating.

\section{Nanoparticle Synthesis Using Glycerol}

Glycerol, an abundant [54] and safe by product from biodiesel production, has gained attention as an alternative sustainable solvent for catalytic reaction and nanomaterials synthesis because of its unique physical and chemical properties, such as high polarity, low toxicity, high boiling point, and biodegradability. The viability of this greener approach using glycerol has been established for the fabrication of $\mathrm{Au}, \mathrm{Pt}$, and Pd nanomaterials under MW irradiation conditions.

\section{Synthesis of Nanoparticles Using Beet Juice}

A facile and benign protocol has been established for the fabrication of hybrid $\mathrm{AgCl} / \mathrm{Ag}$ plasmonic nanoparticles under MW irradiation conditions, Beet juice, an abundant sugar rich bio-renewable agricultural vegetable by product, served as a reducing reagent. This method does not require additional reducing agent or surfactant, and the prepared plasmonic photocatalyst, $\mathrm{AgCl} / \mathrm{Ag}$, is efficient for application under visible light [55].

\section{Conclusion}

The entry of microwave ovens in the chemistry laboratory has made it possible to carry out many transformations with greater efficiency and ease of workup. In recent years, the use of microwave has become very attractive in the field of pharmaceutical science. Microwaveassisted synthesis is a convenient way toward the goal of green chemistry. 
Microwave irradiation can be used in chemical synthesis as a heat source; it is very efficient and can be used to significantly reduce reaction times of numerous synthetically useful chemical transformations. In order to achieve further development in this field, novel instruments, which give rise to reproducible performances and that constitute a minimal hazard should be used instead of the domestic microwave ovens. We believe that in the future many more microwaveassisted reactions will be developed which will simplify time consuming conventional procedures thereby increasing the overall efficiency of the processes and reducing pollution of the environment through the use of solvent free reaction protocols. The exploitation of microwaves for assisting different organic reactions has blossomed into an important tool in synthetic organic chemistry. The future of microwave technology looks bright because of its efficiency and its potential to contribute to clean products.

\section{ORCID}

S. Ravichandran (D) 0000-0001-7281-2778

\section{References}

[1]. P.T. Anastas, J.C. Warner. Green Chemistry, Theory and Practice, Oxford University Press: Oxford, 1998, p 85.
[2]. A.S., Grewal, K. Kumar, S. Redhu, S. Bhardwaj, Int. Res. J. Pharm. App. Sci., 2013, 3, 278-285.

[3]. P. Lidstrom, J. Tierney, B. Watheyb, J. Westmana, Tetrahedron, 2001, 37, 92259283.

[4]. M. Lancaster. Principles of sustainable and green chemistry. Handbook of green chemistry and technology, 2002, pp.10-27.

[5]. M. Kidwai, R. Venkataraman, B. Dave, Green Chem., 2001, 3, 278-279.

[6]. R.S. Varma, R.K. Saini, R. Dahiya, Tetrahedron Lett., 1997, 38, 7823-7824.

[7]. A. Loupy, Microwaves in Organic Synthesis, Wiley-VCH: Weinheim, 2002, p 68.

[8]. P. Lidstrom, J. Tierney, B. Wathey, Tetrahedron, 2001, 57, 9225-9283.

[9]. R. Arun kumar, K. Subramani, S. Ravichandran, Int. J. Chem. Tech. Res., 2010, 2, 278-281.

[10]. S. Ravichandran, Synth. Commun., 2001, 31, 2059-2062.

[11]. S. Ravichandran, Synth. Commun., 2001, 31, 2185-2188.

[12]. R. Sanghi, Resonance, 2000, 5, 77-81.

[13]. R.S. Varma, Tetrahedron, 2002, 58, 1235-1255.

[14]. D.D. Artman, A.W. Grubbs, R.M. Williams, J. Amer. Chem. Soc., 2007, 129, 6336-6342.

[15]. S. Ravichandran, K. Subramani, R. Arun Kumar, Int. J. Chem. Sci., 2008, 6, 1800. 
[16]. C. Zhang, L. Liao, S. Gong, Green Chem., 2007, 9, 303-314.

[17]. S. Sinwell, H. Ritter, Aus. J. Chem., 2007, 60, 729-743.

[18]. R.A. Sheldon, I. Arends, U. Hanefeld, Green Chemistry and Catalysis, Wiley: Wienheim, 2007, 1, p 18.

[19]. P.T. Anastas, J.C. Warner, Green Chemistry: Theory and Practice, Oxford University Press, Oxford, 2000, 2, p 124.

[20]. M. Lancaster, Green Chemistry $3^{\text {rd }}$ Ed: An Introductory Text, Royal Society of Chemistry: Cambridge, 2016, 1, p 58.

[21]. U.J. Joshi, K.M. Gokhale, A.P. Kanitkar, Indian J. Pharm. Edu. Res., 2011, 45, 168-174. [22]. J.H. Clark, D.J. Macquarrie, Handbook of Green Chemistry and Technology, John Wiley \& Sons, 2008, pp.10-27.

[23]. S. Ravichandran, E. Karthikeyan, Int. J. Chem. Tech. Res., 2011, 3, 466-470.

[24]. J.L. Krstenansky, I. Cotterill, Curr Opin Drug Discov Devel, 2000, 3, 454-461.

[25]. B.S. Sekhon, Int. J. PharmTech. Res., 2010, 2, 827-833.

[26]. H. Rajak, P. Mishra, J. Sci. Ind. Res., 2004, 63, 641-654.

[27]. B. Wathey, J. Tierney, P. Lidström, J. Westman, Drug Discov Today, 2002, 7, 373380.

[28]. P. Lidström, J. Tierney, B. Wathey, J. Westman, Tetrahedron, 2001, 57, 92259283.

[29]. E.H. Grant, B.J. Halstead, Chem. Soc.
Rev., 1998, 27, 213-224.

[30]. C.R. Strauss, R.W. Trainor, Aust. J. Chem., 1995, 48, 1665-1692.

[31]. F. Langa, P. Cruz, A. Hoz, A. Diaz-Ortiz, E. Diez-Barra, Contemp. Org. Synth., 1997, 4, 373-386.

[32]. P. Lidström, J. Westman, A. Lewis, Comb. Chem. High Throughput Screen., 2002, 5, 441-458.

[33]. O. Algul, A. Kaessler, Y. Apcin, A. Yilmaz, J. Jose, Molecules, 2008, 13, 736-748.

[34]. M.J. Collins Jr, Future Med. Chem., 2010, 2, 151-155.

[35]. M. Larhed, A. Hallberg, Drug Discov. Today, 2001, 6, 406-416.

[36]. A. Lew, P.O. Krutzik, M.E. Hart, A.R. Chamberlin, J. Comb. Chem., 2002, 4, 95-150. [37]. N.S. Wilson, C.R. Sarko, G.P. Roth, Org. Proc. Res. Dev., 2004, 8, 535-538.

[38]. S.V. Ley, I.R. Baxendale, Nat. Rev. Drug. Discov., 2002, 1, 573-586.

[39]. M. Gaba, N. Dhingra, Indian J. Pharm. Edu. Res., 2011, 45, 175-183.

[40]. I. Montes, D. Sanabria, M. García, J. Fajardo, J. Chem. Edu., 2006, 83, 628-631.

[41]. D. Bogda, J. Pielichowski, A. Borona, Synlett, 1996, 873-874.

[42]. V. Chakraborty, M. Bordoloi, J. Chem. Res. (S), 1999, 118-122.

[43]. S. Ravichandran, E. Karthikeyan, Int. J. ChemTech. Res., 2011, 3, 466-470.

[44]. R. Boscencu, Molecules, 2012, 17, 55925603. 
[45]. M. Baghbanzadeh, L. Carbone, P.D. Cozzoli, C.O. Kappe, Angew. Chem. Int. Ed. Engl., 2011, 50, 11312-11359.

[46]. K.J. Sreeram, M. Nidhin, B.U. Nair, Bull. Mater. Sci, 2008, 31, 937-942.

[47]. V. Polshettiwar, M.N. Nadagouda, R.S.Varma, Aust. J. Chem., 2009, 62, 16-26.

[48]. M. Gupta, B.P. Wakhloo, Arkivoc, 2007, 15, 94-98.

[49]. Z.L. Jiang, Z.W. Feng, X.C. Shen, Chin. Chem. Lett., 2001, 12, 551-554.

[50]. J. Jacob, Int. J. Chem., 2012, 4, 29-43.

[51]. S.L. Petersen, A.P. Tofteng, L.Malik, K.J. Jensen, Chem. Soc. Rev., 2012, 41, 1826.

[52]. R. Deng, Y. Wang, Y. Jiang, Synth. Commun., 1994, 24, 111-115.

[53]. C. Gabriel, S. Gabriel, E.H. Grant, B.S.J. Halstead, P.M. Mingos, Chem. Soc. Rev., 1998,
27, 213-224.

[54]. M.B. Gawande, A.K. Rathi, P.S. Branco, I.D. Nogueira, A. Velhinho, J.J. Shrikhande, U.U. Indulkar, R.V. Jayaram, C.A. Ghumman, N. Bundaleski, O.M. Teodoro, Eur. J., 2012, 18, 12628-12632.

[55]. J. Kou, R.S. Varma, ChemSusChem., 2012, 5, 2435-2441.

[56]. M.A. Surat, S. Jauhari, K.R. Desai, Indian J. Pharm. Edu. Res., 2012, 4, 645.

[57]. Z. Chemat-Djenni, B. Hamada, F. Chemat, Molecules, 2007, 12, 1399-1409. [58]. R.S. Varma, K.P. Naicker, Tetrahedron Lett., 1999, 40, 6177-6180.

[59]. J. Jacob, Int. J. Chem., 2012, 4, 29-43. [60]. A. Madhavi, J. Smita, K.R. Desai, Archiv. Appl. Sci. Res., 2012, 4, 645-661.

How to cite this manuscript: Sumitra Nain, Ruchi Singh, Subramanian Ravichandran*, Importance of Microwave Heating in Organic Synthesis, Adv. J. Chem. A, 2019, 2(2), 94-104. 